\title{
Dinámica de los hongos de Micorriza Arbuscular (MA) en un Humic Dystrudepts sembrado con maíz Zea mays L. y Abonos Verdes (AV)
}

\section{Dynamic of Arbuscular Mycorrhiza (AM) fungus in a Humic Dystrudepts sowed with corn Zea mays L. and Green Compost (GC)}

\author{
A dinâmica de fungos micorrízicos arbusculares (MA) \\ em um Cambissolo Húmicas plantadas com Zea mays $L$. \\ e adubo verde (AV)
}

\author{
Francisco Javier Vélez Zabala1 \& Marina Sánchez de Prager $^{2}$ \\ Facultad de Ciencias Agropecuarias, Universidad Nacional de Colombia \\ Sede Palmira, Valle del Cauca. \\ fjvelezz@unal.edu.co,msanchezpr@unal.edu.co
}

\begin{abstract}
${ }^{1}$ Magíster en Ciencias Agrarias, Ingeniero Agrónomo, Universidad Nacional de Colombia Sede Palmira. Docente, Universidad Nacional Abierta y a Distancia (UNAD); Escuela de Ciencias Agrícolas,

Pecuarias y del Medio Ambiente (ECAPMA). Palmira, Colombia.

${ }_{2}^{2}$ Profesora Titular, Universidad Nacional de Colombia Sede Palmira.
\end{abstract}

\section{Resumen}

Los AV inciden positivamente sobre propiedades físicas, químicas y biológicas del suelo, entre ellas, la expresión de la MA. En un Humic Dystrudepts en zona montañosa de Palmira (Valle) se establecieron cinco tratamientos: Testigo (T), Fertilización de síntesis química industrial (FQ), Abono verde (AV), Compost $(\mathrm{C}) \mathrm{y}$, Abono verde+Compost $(\mathrm{AV}+\mathrm{C})$, en un arreglo de bloques completos al azar, con tres repeticiones. Como AV se intercalaron Canavalia ensiformis L. y Axonopus scoparius F., se cosecharon en prefloración de la leguminosa, incorporaron y se hizo una primera siembra de maíz, variedad ICA V-354, monitoreado durante 150 días, hasta época de llenado de mazorca, cuando se evaluó longitud de micelio externo total, LMET (Técnica del filtro de membrana y método del intercepto de Miller and Jastrow), aquel vivo y activo, LMEV (succinato-deshidrogenasa, Hamel et al., 1990) y, porcentaje de colonización por arbúsculos (Phillips y Hayman, 1980, descrita por Sieverding, 1983 y adaptada para este cultivo, Sánchez et al., 2010). El análisis de varianza (software $\mathrm{SAS} \otimes$ versión 9.1.3) mostró que LMET, LMEV y \% de arbúsculos presentaron diferencias altamente significativas entre tratamientos. Mayor LMET, coincidió con incrementos en LMEV, colonización por arbúsculos $y$, se presentó en $A V+C$, seguido por $A V, C$ y $T$, que no variaron entre sí, y, los más bajos en FQ. La coincidencia de estas tres variables, indicaría que a través de LMET y especialmente, LMEV se está absorbiendo y transportando $\mathrm{P}$ al interior de las plantas, y este suministro, coincidente con mayor $\%$ de colonización por arbúsculos, señala la ruta del $\mathrm{P}$ en la conjunción suelo-planta-fertilización orgánica, con sus consecuencias sobre la economía del agroecosistema y del agricultor. 
Palabras clave: micorriza arbuscular (MA); longitud de micelio externo total (LMET); longitud de micelio externo vivo (LMEV), abonos verdes (AV).

\section{Abstract}

Green Compost (GC) have a positive impact on physical, chemical and biological properties of the soil, among them, the expression of the MA. In a Humic Dystrudepts in a mountainous area of Palmira (Valle) five treatments were established: $(T)$, fertilization of insdustrial chemical synthesis (FQ), green manure (AV), Compost ${ }^{(C)}$ and green manure compost (AVC), in a settlement of randomized complete blocks with three replications. As GC intermingled Canavalia ensiformis L. and Axonopus scoparius F., were harvested in prefloration of the legume, incorporated and became the first planting of corn, variety ICA $\mathrm{V}$-354, monitored during 150 days, until time of fill of cob, when assessed length total external mycelium, LMET (Technical of the membrane filter method and the intercepted of Miller and Jastrow), that alive and active LMEV (succinate dehydrogenase, Hamel et al., 1990) and percentage of colonization by branch out manifold (Phillips and Hayman, 1980, described by Sieverding, 1983 and adapted for this crop, Sanchez et al. , 2010). The analysis of variance (SAS $®$ software version 9.1.3) showed that LMET, LMEV and $\%$ of branch out manifold showed highly significant differences between treatments. Greater LMET, coincided with increases in LMEV, colonization by branch out manifold and is presented in GC C, followed by $\mathrm{AV}, \mathrm{C}$ and $\mathrm{T}$, which did not vary among themselves, and, the lowest in CF. The coincidence of these three variables, would indicate that through LMET and especially, LMEV is absorbing and transporting $P$ to the inside of the plants, and this supply, coinciding with higher $\%$ of colonization by branch out manifold, traces the path of the conjunction $\mathrm{P}$ in the soil-plantorganic fertilization, with their consequences on the economy of the agroecosystem and farmer.

Key-words: arbuscular mycorrhiza (MA); length of total external mycelium (LMET); length of external mycelium alive (LMEV), green compost (GC).

\section{Resumo}

O impacto positivo de adubos verdes (AV) em propriedades físicas, químicas e biológicas do solo, incluindo a expressão de Micorriza Arbuscular (MA) em um Humic Dystrudepts numa área montanhosa do município de Palmira na Colômbia foram estabelecidos cinco tratamentos: Testemunha $(T)$, Fertilização síntese química industrial (FQ), adubação verde (AV), compostagem (C) e adubo verde + Compostagem $(\mathrm{AV}+\mathrm{C})$, em um arranjo de blocos ao acaso com três repetições. Com AV foram intercaladas Canavalia ensiformis L. e Axonopusscoparius F.colheitas na prefloração das leguminosas, incorporaram-se e se fez o primeiro plantio de milho, A variedade ICA V-354 foi monitorada por 150 dias, até à hora de encher espiga, quando foi avaliado o comprimento total de micélio externo-LMET (técnica de membrana filtrante e Miller e Jastrow método de interceptação), vivo e ativo- LMEV (succinato desidrogenase, Hamelet al., 1990) e porcentagem de colonização foi avaliada por arbúsculos (Phillips e Hayman, 1980, descrita por Sieverding, 1983 e adaptado para esta safra, Sánchez et al., 2010). A análise de variância (software SAS versão 9.1.3) mostrou que LMET, LMEV e \% de arbúsculos mostraram diferenças altamente significativas entre os tratamentos. Os valores mais altos de LMET, coincidiram com o aumento da LMEV, Apresentou-se que a colonização por arbúsculos em $\mathrm{AV}+\mathrm{C}$, seguido por AV, C e T, não diferem uns dos outros, e os mais baixos na FQ. A coincidência de estas três variáveis, indica que através de LMET e especialmente LMEV está sendo absorvido e transportado fosforo $(P)$ no interior das plantas, e este fornecimento, pela sua vez coincide com uma Maior $\%$ de colonização por arbúsculos, sinalizando a rota do $\mathrm{P}$, em conjunto solo-planta- fertilização orgânica, com o seu impacto sobre a economia do agroecossistema e agricultor.

Palavras-chave: micorriza Arbuscular (MA); Comprimento total de micélio externo (LMET); Comprimento vivo micélio externo (LMEV), adubação verde (AV). 


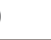

\section{Introducción}

Debido al alto costo de los insumos de síntesis industrial, a las evidencias de contaminación ambiental y degradación de suelos, se hace necesario optar por alternativas para disminuir o sustituir estos insumos. En la búsqueda de soluciones se han planteado prácticas agroecológicas como el uso de los AV.

Los AV constituyen una práctica que se realiza por distintas comunidades campesinas desde hace tiempo. Consiste en sembrar especies que en poco tiempo desarrollan biomasa, la cual se incorpora al suelo para proveer nutrientes en un cultivo posterior. Las plantas más utilizadas corresponden a leguminosas y gramíneas, aunque pueden emplearse otras especies.

Los AV influyen en características físicas del suelo así: mejora la retención de humedad y facilita la penetración de capas con raíces profundas (Astier et al., 2007, Zhang et al., 2007). En cuanto a características químicas: aumenta la materia orgánica, el retorno de $\mathrm{N}$ a través de la fijación biológica de $\mathrm{N}_{2}$, disminuye el lavado de nutrientes, puede incrementar el pH, la acumulación del $\mathrm{C}$ orgánico y la mineralización del $\mathrm{N}$ y $\mathrm{P}$ orgánico, pudiendo complementar o sustituir fertilizantes de síntesis industrial (Prager et al., 2000; Randhawa et al., 2005; Hoon Lee et al., 2010).

Los AV están destinados a ser fuente de materia orgánica fácilmente degradable por la actividad de los organismos del suelo. La actividad microbiana en la rizosfera de las plantas es la encargada de mineralizar estos materiales orgánicos y colocarlos a disponibilidad de las plantas que se siembran después de los AV (Gómez, 2000; Prager et al., 2002; Sánchez de P. et al., 2007). En esta actividad participan diversidad de microorganismos entre ellos bacterias, hongos y dentro de estos un grupo muy especial que forman una simbiosis los hongos formadores de micorriza arbuscular (HMA), que participan activamente en el mantenimiento de la fertilidad y salud del suelo (Jeffries et al., 2003; Molina, 2005).
Dados sus efectos sobre la nutrición de la planta, el estudio de los HMA cobra importancia, en particular en los suelos tropicales. La presencia de zonas de agotamiento de nutrientes, especialmente de aquellos con baja disponibilidad, ocurre en aproximadamente el $36 \%$ de los suelos tropicales, de los cuales, el $23 \%$ presentan alta fijación de P (Sánchez \& Logan, 1992; Hue et al., 1994; Molina, 2005). En estos suelos el $P$ está fuertemente retenido por procesos de adsorción y precipitación, debido a la presencia de sesquióxidos de hierro y aluminio y materiales alófanicos (Gijsman \& Sanz, 1998; Rao et al., 1999; Bühler et al., 2002). Entonces, la disponibilidad de este elemento se torna limitante en los procesos de producción.

Los HMA al formar la simbiosis con la planta llamada micorriza arbuscular (MA) tornan eficiente la absorción del $\mathrm{P}$ y contribuyen a través de sus componentes como el micelio externo (ME) y micelio interno (MI) a que la zona de agotamiento del nutriente que se presenta en el suelo sea superada con creces (Sánchez de P. et al., 2007).

El estudio del efecto de los AV y MA se ha concentrado en la disponibilidad de $\mathrm{P}$ para las plantas y sus efectos en los rendimientos, sin embargo, aún falta comprender la participación de los componentes de los HMA (micelio y arbúsculos) como compartimentos a través de los cuales circula el nutriente en la interfase suelo-planta. De allí que esta investigación aborde esta temática.

El objetivo general de este trabajo fue evaluar el efecto de la adición de abonos verdes y hongos formadores de micorriza, estrechamente relacionados con absorción de nutrientes en un ciclo de siembra de maíz Zea mays $L$. 


\section{Materiales y métodos}

\section{Localización del ensayo}

El presente trabajo se desarrolló en el marco del proyecto "Los Abonos Verdes como Estrategia Agroecológica y Ambiental en Agroecosistemas del Valle del Cauca" que adelanta el grupo de investigación en Agroecología de la Universidad Nacional de Colombia. Se realizó entre octubre de 2009 y septiembre de 2010 en el departamento de Valle del Cauca, coordenadas geográficas

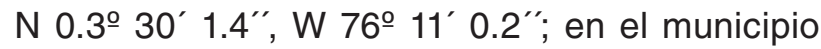
de Palmira, corregimiento Chontaduro, finca las Flores a una altitud de 1713 m.s.n.m., con temperatura promedio $20.8{ }^{\circ} \mathrm{C}$, precipitación media $139.6 \mathrm{~mm}$, y humedad relativa media anual del 74.4\% (CVC, 2011).

\section{Tratamientos, descripción del ensayo y parámetros a evaluar}

Para cumplir con los objetivos propuestos, se evaluaron algunas propiedades físico-químicas del suelo a estudiar mediante metodologías resumidas en la Tabla 1.

Los tratamientos evaluados se especifican en la Tabla 2. El tratamiento de AV incluyó una mezcla de una leguminosa y una gramínea, comparados con fertilización de síntesis industrial, compost como opción de materia orgánica, la mezcla de ambos y un testigo absoluto, que imitó la forma como cotidianamente los agricultores siembran en la zona.

Se utilizó un diseño experimental de bloques completos al azar BCA. La unidad experimental del ensayo correspondió a una parcela de 2.5 $\mathrm{m}$ de ancho por $4 \mathrm{~m}$ de largo, con un área total de $10 \mathrm{~m}^{2}$.
Tabla 1. Metodologías usadas en el análisis químico del suelo estudiado.

\begin{tabular}{lc}
\hline \multicolumn{1}{c}{$\begin{array}{c}\text { Parámetro } \\
\text { Evaluado }\end{array}$} & $\begin{array}{c}\text { Método analítico (*) } \\
\text { pH agua 1:1 }\end{array}$ \\
\hline ElC & $\begin{array}{c}\text { Potenciómetro } \\
\text { Amonio acetato }\end{array}$ \\
\hline Materia orgánica & Espectrometría \\
\hline Fosforo Bray II & Espectrometría \\
\hline Potasio inter. & Aa \\
\hline Calcio inter. & Aa \\
\hline Magnesio inter. & Aa \\
\hline Aluminio Cambiable & $(\mathrm{KCl} 1 \mathrm{M})$ \\
\hline Sodio inter. & $\mathrm{Aa}$ \\
\hline Azufre extractable & $($ Fosfato Ca) \\
\hline Boro & Espectrometría \\
\hline Hierro & $\mathrm{DA}, \mathrm{Aa}$ \\
\hline Manganeso & $\mathrm{DA}, \mathrm{Aa}$ \\
\hline Cobre & $\mathrm{DA}, \mathrm{Aa}$ \\
\hline Zinc & $\mathrm{DA}, \mathrm{Aa}$ \\
\hline
\end{tabular}

DA (Doble acido), Aa (Absorción atómica)

*Fuente de información: CIAT, 2006.

Tabla 2. Tratamientos utilizados en la investigación.

\section{Tratamiento Descripción}

T1 Testigo (T) Cultivo de maíz con manejo local.

T2

Fertilización químico (FQ)

Maíz con fertilización química

T3 Abonos verdes (AV)

Maíz con incorporación de AV

T4 Compost (C)

Maíz + compost

Abonos verdes +
Compost $(\mathrm{AV}+\mathrm{C})$

Maíz con AV y compost 


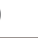

\section{Abonos verdes}

El AV resultó de la mezcla de la leguminosa Canavalia (Canavalia ensiformis L.) y la gramínea, Pasto Imperial (Axonopus scoparius F.). Al inicio del ensayo, los AV se sembraron en las unidades experimentales correspondientes y se dejaron crecer durante aproximadamente 3 meses, hasta la época de prefloración de la leguminosa, después se cortó e incorporó en los respectivos tratamientos.

La siembra de los AV se efectuó con distancias de $0.5 \mathrm{~m}$ entre surcos para ambas especies. Para $C$. ensiformis la distancia entre plantas fue de $0.15 \mathrm{~m}$. con densidad de siembra de 133.332 plantas ha-1. Para A. scoparius la distancia entre plantas fue de $0.20 \mathrm{~m}$. con una densidad de 100.000 plantas ha-1. Estos se establecieron en los tratamientos bajo esta práctica.

\section{Compost}

Se escogió el compost elaborado en el Bolo en la finca de Elizabeth Martínez, al cual se realizaron análisis químico de $\mathrm{C}$-total, $\mathrm{N}$-Total, $\mathrm{P}$-total, $\mathrm{K}$, Ca, Mg, S-total, B, Fe, Mn, Cu, Zn (Benton, 1993; Mackean, 1993). Las evaluaciones del suelo y del compost se realizaron en el laboratorio de servicios analíticos CIAT.

\section{Maíz}

El material vegetal que se utilizó fue la variedad ICA V-354, de color blanco. Según indicaciones del productor obtenidas, este material vegetal alcanza rendimientos de 4.500 a $5.500 \mathrm{~kg} \mathrm{ha}^{-1}$ tiene un rango de adaptación de 0 a 1.600 m.s.n.m. y un periodo vegetativo de 160 a 170 días en zonas de ladera.

Al día siguiente de incorporados los AV, el maíz se sembró por el método de siembra directa a razón de 2 semillas pre germinadas/sitio. La distancia de siembra utilizada fue de $0.80 \mathrm{~m}$ entre surcos y $0.25 \mathrm{~m}$ entre plantas, para densidad de 50.000 plantas ha-1, la cual emplean los agricultores en esta zona. Cada unidad experimental comprendió 5 surcos, y se separaron entre ellas por una distancia de $0.6 \mathrm{~m}$ y cada bloque o repetición se separó por una distancia de $1.4 \mathrm{~m}$. El área total que ocupó el ensayo fue de aproximadamente $225 \mathrm{~m}^{2}$.

\section{Fertilización}

Para los respectivos tratamientos se utilizó NPK distribuido en 3 etapas de aplicación: la primera aplicación se realizo en abril 28 se aplicó 290 g/ parcela de NPK $4.16 \mathrm{~g} / \mathrm{sitio}$, la segunda aplicación en mayo 20: urea $5 \mathrm{~g}+\mathrm{KCl} 5 \mathrm{~g} /$ planta, la tercera aplicación en junio $10 \mathrm{5g}$ Urea/planta y la cuarta aplicación: el 28 de junio se aplicó a todo el cultivo $\mathrm{SHI}-3$ + elementos menores $100 \mathrm{~cm}^{3}+5 \mathrm{~g}$ elementos menores/2OL de $\mathrm{H}_{2} \mathrm{O}$.

\section{Variables a evaluar}

Aquellas correspondientes a los análisis físicoquímicos del suelo y compost como marco que permita explicar los resultados obtenidos en la variable biológica.

Para las variables biológicas se efectuaron tres muestreos: antes de la siembra del maíz (asm), a los 36 días después de siembra (36 ddsm), época de llenado de mazorca (90 ddsm). Las variables estimadas en cada muestreo fueron: longitud de ME (LME), ME vivo y activo (MEV), porcentaje de colonización por arbúsculos (\% A).

\section{Longitud de micelio externo (LME)}

Para la extracción y cuantificación de LME se empleó la técnica del filtro de membrana (Miller \& Jastrow, 1990, modificada por Torres, 2000 y Reyes, 2001). LME se estimó por el método del campo de 
intercepto "grid line" y los cálculos se realizaron por el método de Tennant (1975), descritos y adaptados por Torres (2000) y Zárate (2006), considerando las dimensiones específicas para el microscopio y equipo de filtro usado.

Este proceso se inició con la dispersión de $5 \mathrm{~g}$ de suelo en hexametafosfato de sodio al $5 \%$, posterior lavado con $500 \mathrm{ml}$ de agua deionizada usando tamiz de $25 \mathrm{~m}$, dilución de la muestra para facilitar su observación al microscopio y finalmente recolección del micelio en un equipo de filtración al vacío con filtros de nitrocelulosa de $1.2 \mathrm{~m}$, con posterior tinción del micelio con tinta Sheaffer al 5\% para facilitar la cuantificación de LME en términos de m/gss (metodología descrita por Sánchez de P. et al., 2010).

\section{Longitud de micelio externo vivo y activo (LMEV)}

Esta tinción que permitió estimar la LMEV, es decir, aquel que participa activamente en la absorción y transporte de $\mathrm{P}$, se ha basado en la coloración de espacios celulares específicos en los cuales, la succinato - deshidrogenasa (complejo enzimático que hace parte del ciclo de Krebs) al reaccionar con una solución incubadora que contiene succinato de sodio y azul de tetrazolio (Sánchez de P. et al., 2010), se torna de coloración rojiza e indican que allí esta ocurriendo transporte de P. Se realizó siguiendo la metodología de Hamel et al. (1990), modificada en el Instituto Canario de Investigaciones Agrarias, Santa Cruz de Tenerife, España 2007.

\section{Porcentaje de colonización total por HMA y por arbúsculos}

Se siguió la metodología descrita por Sieverding (1983), Sánchez et al. (2010) con las adaptaciones requeridas para el cultivo de maíz, así:
Se separaron del suelo las raíces delgadas de las plantas, mediante lavados continuos. Se colocaron en tubos de ensayo, se añadió $\mathrm{KOH}$ al $10 \%$ y se incubaron en un baño María a $80^{\circ} \mathrm{C}$ por 10 minutos. Las raíces se lavaron con agua y se aclararon con $\mathrm{HCl}$ al $10 \%$ por 5 minutos. Se lavaron nuevamente con agua. Posteriormente se agregó tinta Sheaffer color azul al $5 \%$ en vinagre por 3 minutos y se incubó nuevamente a baño María a $80^{\circ} \mathrm{C}$. Una vez realizada la tinción se las dispuso en una caja de Petri y se agregó glicerina al 50\% para conservarlas.

Las raíces previamente teñidas se cortaron en trozos de $1.5 \mathrm{~cm}$ aproximadamente y se ubicaron 10 de ellos por portaobjetos, luego se agregó glicerina $(50 \%)$ y se cubrió con cubreobjetos.

Se hicieron tres repeticiones/parcela, para una evaluación total de $135 \mathrm{~cm}$ de raíces/tratamiento (30 trozos/repetición con 3 repeticiones/tratamiento $x 1.5 \mathrm{~cm} /$ trozo). Con un aumento de $40 \mathrm{X}$, se leyeron 24 campos en promedio por lámina distribuidos en recorridos longitudinales, anotando como positivo la presencia de cualquier estructura micorrícica encontrada en cada uno de los trozos de raíz. La sumatoria de los campos positivos se lleva a la ecuación 1:

\section{$\%$ de Colonización total por HMA =} \# de campos colonizados observados X 100

\# total de campos observados

\section{Análisis de resultados}

Las variables de respuesta se analizaron con el paquete estadístico SAS (software SAS® versión 9.1.3, 2009). En los casos en los cuales se encontraron diferencias significativas $(P \leq 0.05)$ entre fuentes de variación se hicieron pruebas de comparación de medias Duncan y Tukey. Para estimar las correlaciones entre las variables se calculó el coeficiente de Pearson con nivel de significancia $\mathrm{P} \leq 0.05$. 


\section{Resultados y discusión}

\section{Algunas características físico-químicas del suelo estudiado}

La caracterización taxonómica del suelo de la finca Las Flores, la efectuó la doctora Yolanda Rubiano. Este es un Inceptisol, con perfil Humic dystrudepts (USDA, 2010), descrito así: "Presenta un epipedón úmbrico, que descansa sobre un endopedón cámbico, con predominio de arcillas 1:1. La saturación de bases es mayor del $50 \%$, el régimen de humedad es údico influenciado por una precipitación media anual de $139.6 \mathrm{~mm}$. La textura del suelo dentro de sus primeros $30 \mathrm{~cm}$ es arcillo limoso y contiene fragmentos de grava fina. Presenta una pendiente del $30 \%$ clasificada como escarpada".

Respecto a las características químicas (Tabla 3) el pH es moderadamente ácido; con altos contenido de materia orgánica, $\mathrm{CIC}$, calcio, magnesio, potasio, boro y zinc. El fósforo y cobre disponibles se catalogaron como medios y bajo el hierro (Menjívar, 2010). El manganeso se consideró excesivo con $174.04 \mathrm{mg} \mathrm{kg}^{-1}$; sin embargo, debido al $\mathrm{pH}$, este elemento no se expresó en problemas de toxicidad para el cultivo de maíz (Menjivar, 2010).

Tabla 3. Algunas características químicas iníciales del suelo de la finca Las Flores.

\begin{tabular}{|c|c|c|}
\hline Elementos & Unidades & Valores \\
\hline $\mathrm{pH}$ & & 5,6 \\
\hline MO & $\mathrm{g} \mathrm{kg}^{-1}$ & 74,7 \\
\hline P-Bray II & $\mathrm{mg} \mathrm{kg}^{-1}$ & 11,4 \\
\hline $\mathbf{K}$ & $\mathrm{cmol} \mathrm{kg}^{-1}$ & 0,8 \\
\hline $\mathrm{Ca}$ & $\mathrm{cmol} \mathrm{kg}^{-1}$ & 10,6 \\
\hline Mg & $\mathrm{cmol} \mathrm{kg}^{-1}$ & 6,1 \\
\hline Al & $\mathrm{cmol} \mathrm{kg-}^{-1}$ & 0 \\
\hline $\mathrm{Na}$ & $\mathrm{cmol} \mathrm{kg}^{-1}$ & 0,05 \\
\hline $\mathrm{CIC}$ & $\mathrm{cmol} \mathrm{kg}^{-1}$ & 34,3 \\
\hline $\mathrm{S}$ & $\mathrm{mg} \mathrm{kg}^{-1}$ & 53,4 \\
\hline B & $\mathrm{mg} \mathrm{kg}^{-1}$ & 0,7 \\
\hline $\mathrm{Fe}$ & $\mathrm{mg} \mathrm{kg}^{-1}$ & 5,1 \\
\hline Mn & $\mathrm{mg} \mathrm{kg}^{-1}$ & 174,04 \\
\hline $\mathrm{Cu}$ & $\mathrm{mg} \mathrm{kg}^{-1}$ & 1,9 \\
\hline Zn & $\mathrm{mg} \mathrm{kg}^{-1}$ & 5.5 \\
\hline
\end{tabular}

Fuente: Laboratorio de servicios Analíticos CIAT (2010). 


\section{Propiedades químicas del compost}

\section{Algunas características químicas del Compost y Relación C/N de los Abonos verdes incorporados}

El análisis del compost utilizado en este ensayo (Tabla 4) mostró una relación $\mathrm{C} / \mathrm{N}$ de 24.9 , considerada adecuada para una enmienda o acondicionador a aplicar en esta zona. Sin embargo, el alto contenido de manganeso, posiblemente por el origen del material inicial compostado, hace que los riesgos de exceso de manganeso se incrementen, dado el análisis del suelo que mostraron que este elemento ya aparecía alto. Indagando en la historia del lote, se encontró que en este sitio, hace algunos años escurrían lixiviados de una porqueriza establecida en la parte alta.

El contenido de Nitrógeno hace que a esta enmienda o acondicionador se considere como un "material frío" sujeto a las condiciones de temperatura de la zona, las cuales durante el ensayo oscilaron entre 19 y $23^{\circ} \mathrm{C}$. El resto de nutrientes aportados por el material se calificaron como adecuados.

En el caso de los AV, sólo se analizó la relación $\mathrm{C} / \mathrm{N}$, la cual fue de 17.2, considerada óptima para un suministro rápido de nutrientes (Prager et al. 2000), en especial cuando el análisis de nitrógeno de la mezcla de los dos materiales arrojó un contenido de 3.1\% (Laboratorio de Nutrición Animal, 2010). Nuevamente la temperatura del suelo influyó en la tasa de mineralización de este material, el cual se descompuso rápidamente, al provenir de una leguminosa joven en prefloración.
Tabla 4. Algunas características químicas del compost utilizado en la investigación.

\begin{tabular}{ccc}
\hline Elementos & Unidades & Valores \\
\hline C-Total & $\mathrm{g} \mathrm{kg}^{-1}$ & 185.57 \\
N-Total & $\mathrm{g} \mathrm{kg}^{-1}$ & 7.44 \\
Relación C/N & & 24,94 \\
\hline P-Total & $\mathrm{g} \mathrm{kg}^{-1}$ & 8.74 \\
\hline K & $\mathrm{g} \mathrm{kg}^{-1}$ & 10.92 \\
\hline Ca & $\mathrm{g} \mathrm{kg}^{-1}$ & 83.49 \\
\hline Mg & $\mathrm{g} \mathrm{kg}^{-1}$ & 9.91 \\
\hline S-Total & $\mathrm{g} \mathrm{kg}^{-1}$ & 3.50 \\
\hline B & $\mathrm{mg} \mathrm{kg}^{-1}$ & 37.87 \\
\hline Fe & $\mathrm{mg} \mathrm{kg}^{-1}$ & 91.58 \\
\hline Mn & $\mathrm{mg} \mathrm{kg}^{-1}$ & 519.18 \\
\hline Cu & $\mathrm{mg} \mathrm{kg}^{-1}$ & 52.16 \\
\hline Zn & $\mathrm{mg} \mathrm{kg}^{-1}$ & 142.79 \\
\hline
\end{tabular}

Fuente: Laboratorio de servicios Analíticos CIAT 2010.

\section{Longitud de micelio externo total (LMET)}

El análisis de varianza indicó que la LME presentó diferencias altamente significativas por efecto de los tratamientos, a través del ensayo. En los tratamientos donde se adicionaron materiales orgánicos: T5, T4, T3, se estimuló LME, en comparación con el testigo (T1) y la fertilización química de síntesis industrial (FQ), donde se presentó la menor longitud (Figura 1). 


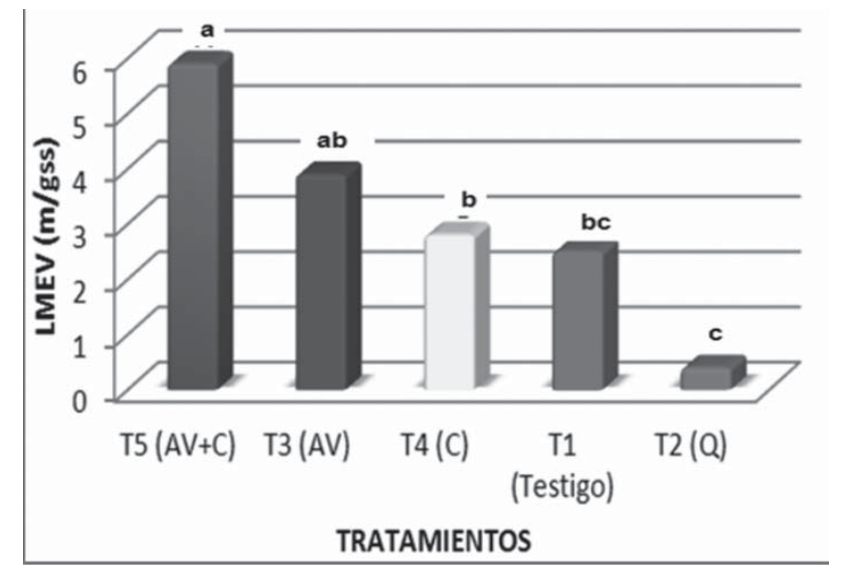

Los valores para LME oscilaron entre 2.6 y 11.7 $\mathrm{m} / \mathrm{gss}$ de suelo, inferiores a los registrados en otras investigaciones, realizadas bajo condiciones de invernadero, en plazos de tiempo cortos y con adiciones de algún tipo de fertilización. El rango registrado en otros ensayos realizados en campo en Colombia ha variado entre $17-20 \mathrm{~m} /$ gss y 18.4-20.2 m/gss en los primeros $10 \mathrm{~cm}$ del suelo (Torres, 2000 y Reyes 2001; Navia, 2006 y Sánchez de P. et al., 2007, respectivamente). Lo anterior puede explicarse por los altos contenidos de fósforo en el suelo utilizado en esta investigación (11.4 mg kg-1), pues como se ha comprobado en otros trabajos, existe una correlación negativa entre este nutriente y LME (Reyes, 2001; Navia, 2006; Zarate, 2006; Sánchez de P. et al., 2007).

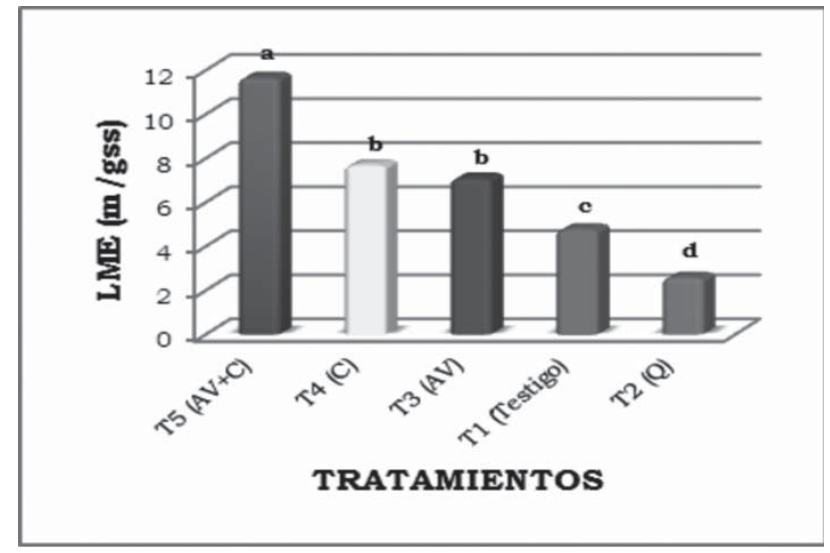

Figura 1. Efecto de tratamientos sobre la LME Total (m/gss) de HMA asociado a plantas de maíz blanco (Zea Mayz). Letras diferentes dentro de cada tratamiento, indican diferencia significativa - Duncan $5 \%$.

\section{Longitud de Micelio externo vivo y activo (LMEV)}

La longitud del micelio externo activo (LMEV) se analizó en la época de llenado de grano del maíz (90 DDS) y se observó que hubo diferencias altamente significativas entre tratamientos. Las mayores diferencias en LMEV se presentaron en $A V+C$ y AV, que difirieron de C, T y FQ (Figura 2). En AV se estimaron $3.9 \mathrm{~m} / \mathrm{gss}$ de LMEV, casi diez veces más longitud que en $Q(0.4 \mathrm{~m} / \mathrm{gss})$.
Figura 2. Efecto de tratamientos sobre la LMEV (m/gss) de HMA asociado a plantas de maíz blanco (Zea Mayz), en época de llenado de mazorca. Letras diferentes dentro de cada tratamiento, indican diferencia significativa - Duncan 5\%.

Con la tinción vital, además de obtener información sobre cuánto de la longitud del micelio total de HMA está participando activamente en la absorción de $\mathrm{P}$, también, varios autores señalan que, puede indicar los cambios que ocurren en el suelo cuando se aplican en los agroecosistemas altas cantidades de fertilizantes de síntesis química industrial. Normalmente, con altas aplicaciones de fertilizantes, el micelio total de HMA disminuye significativamente, pero el micelio activo es más sensible a detectar esta situación, pues se afecta en mayor escala (Sánchez de P., 1999, 2003; Sánchez de P. et al., 2007, 2010). En esta investigación se corroboraron estas afirmaciones: La LME total varió de $11.7 \mathrm{~m} / \mathrm{gss}(\mathrm{AV}+\mathrm{C})$, y $7.1 \mathrm{~m} / \mathrm{gss}(\mathrm{AV}$ ) a $2.7 \mathrm{~m} / \mathrm{gss}$ (en FQ). La LMEV pasó de 5.9 y $3.9 \mathrm{~m} / \mathrm{gss}$ ( $\mathrm{AV}+\mathrm{C}$ y $\mathrm{AV}$, respectivamente) a $0.4 \mathrm{~m} / \mathrm{gss}$ (en $F Q$ ).

En la presente investigación, al momento de llenado de grano de mazorca de maíz (120 DDS), en $A V+C$, de la $L M E$ total, el $50,4 \%$ estaba participando en la absorción de $\mathrm{P}$ (LMEV), mientras que en FQ sólo lo hacía el 14,8\%. Esto, coincide con registros acerca de inhibición en la eficiencia de la actividad de los HMA ante la disponibilidad de $P$ vía fertilizantes de síntesis química industrial (Sánchez de P. et al., 2007, 2010). 


\section{Porcentaje de colonización por arbúsculos}

El análisis de varianza señala diferencias altamente significativas entre tratamientos. El orden del porcentaje de colonización por arbúsculos fue totalmente coherente con los resultados encontrados en LMEV (Figura 3). La discusión adelantada anteriormente se ciñe a esta variable, es decir, que la disponibilidad de $\mathrm{P}$ que se logra a través de la fertilización de síntesis industrial, inhibe los efectos nutricionales de transporte de $P$, que naturalmente se pueden obtener a través de la actividad de los HMA (Sánchez de P. et al., 2007, 2010).

Esta coherencia mostraría una relación de sentido común, pues se podría esperar que el micelio activo de los HMA, tome el P inicialmente del suelo (MEV), lo transporte al interior de las células (micelio interno vivo) y éste, posteriormente se intercambie con las plantas, precisamente en los arbúsculos, señalados como los sitios de máxima actividad metabólica de carácter nutricional (Sánchez de P. et al., 2007; González et al., 2008; Sánchez de P. et al., 2010b).

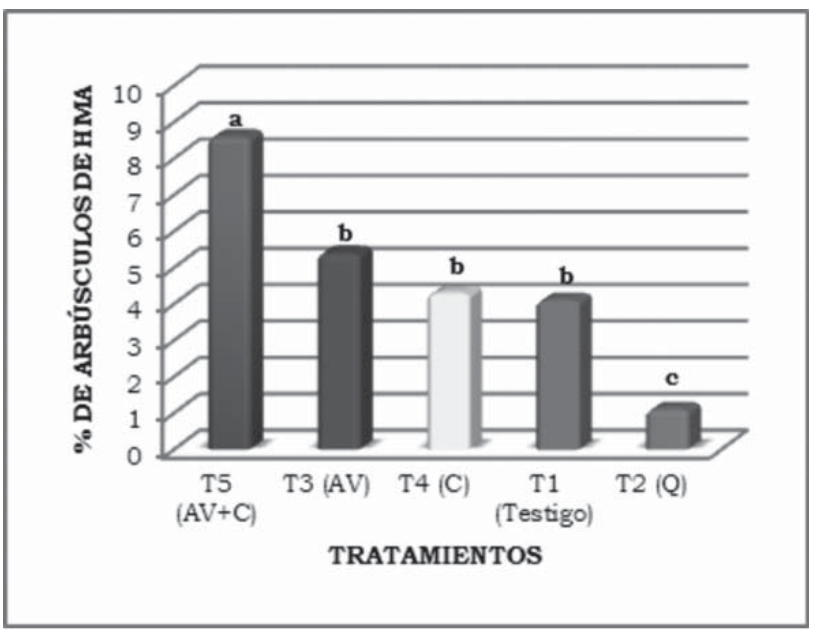

Figura 3. Efecto de tratamientos sobre el porcentaje de arbúsculos de HMA asociado a plantas de maíz blanco (Zea Mayz) en época de llenado de mazorca. Letras diferentes dentro de cada tratamiento, indican diferencia significativa - Duncan $5 \%$.

Los componentes de los HMA están íntimamente relacionados entre sí, lo cual desde el sentido común se podría esperar y lo corrobora el análisis estadístico.
Así, cuando hay mayor LME, se incrementa la colonización por arbúsculos al interior de las células corticales y cuando hay mayor LME, también se detecta mayor LMEV, es decir, aquel que está absorbiendo y transportando $\mathrm{P}$ al interior de las plantas, coincidente con mayor \% de colonización por arbúsculos.

La mayor expresión de las variables biológicas evaluadas LMET, LMEV y Arbúsculos se encuentran con el uso de los materiales orgánicos en mezcla e individuales, mientras que en $\mathrm{T}$ disminuyen y aún más en $F Q$, lo cual permite afirmar que los materiales orgánicos estimulan estas variables, mientras que los fertilizantes de síntesis química industrial las deprimen.

Dentro de la visión de resiliencia y sostenibilidad como dos de los pilares básicos de la Agroecología (Altieri, 2012), la conservación y utilización de los recursos microbiológicos y simbiosis como es el caso de las MA, es fundamental para una agricultura y suelos con futuro. En la medida que la práctica agronómica que empleamos, en este caso $F Q$, o que dejamos de implementar (T), inhibe o disminuye la participación del componente biológico en la fertilidad y conservación del suelo, estamos afectando su productividad y resiliencia a largo plazo (Sánchez de P. et al. 2007).

\section{Conclusiones}

En el suelo Humic Dystrudepts localizado en El Mesón, Finca Las Flores, la mayor expresión de los componentes de HMA: longitud y actividad de micelio externo, micelio externo vivo y porcentaje de colonización por arbúsculos, en el sistema radical del cultivo del maíz blanco ICA V-354, ocurrió con la adición de abonos verdes en mezcla con compost $(\mathrm{AV}+\mathrm{C})$, variables que fueron inhibidas por la fertilización de síntesis química industrial (FQ).

Se encontró una relación directa entre los componentes de HMA evaluados, que llevan a considerar que entre mayor sea la longitud de micelio externo total, mayor puede ser la absorción de $\mathrm{P}$ que ocurre vía micorriza arbuscular. 


\section{Literatura citada}

1. Altieri, M. \& Nicholls, C., (2012). Biodiversidad y manejo de plagas en agroecosistemas. Perspectivas agroecológicas 2. Editorial Icaria. Barcelona, España. 247 p.

2. Astier, M., Maass, J., Etchevers, B., Peña, J. \& González, F. (2007) Short-term green manure and tillage management effects on maize yield and soil quality in an Andisol. EN: Soil and Tillage Research, no. 88. p 153-159.

3. CORPORACION AUTONOMA REGIONAL DEL VALLE DEL CAUCA CVC. Boletín Hidroclimatológico- Año 2011. [En Línea] Cuenca Río Nima, Estación San Emigdio, Municipio de Palmira. Recuperado de: http://www. cvc.gov.co/vsm38cvc/data/RecursoHidrico/aplicativos/ Climatologia/isohietas_Anuales.php

4. Gómez Z. J. (2000). La materia orgánica en los agroecosistemas. Universidad Nacional de Colombia Sede Palmira. 70p.

5. Mackean, S. Manual de suelos y plantas. CIAT, (1993). $120 \mathrm{p}$.

6. Menjívar, J. (2010). Química y Fertilidad de Suelos [diapositivas]. Universidad Nacional de Colombia Sede Palmira, 23 diapositivas.

7. Miller, R. M. \& Jastrow, J. D. (1990). Mycorrhizal fungi influence soil structure. En: Kapulnik, Y. \& Douds, D. D. (eds). Arbuscular Mycorrhizas: Physiology and Function. Kluwer Academic Publishers. London. 3 - 18 p.

8. Navia J. (2006). Impacto de aportes superficiales de biomasa vegetal de diferente calidad sobre poblaciones nativas de hongos formadores de micorriza arbuscular (HMA), rizobios y nematodos, en un suelo agrícola de Santander de Quilichao (Departamento del Cauca). Tesis de doctorado en Ciencias Agropecuarias. Universidad Nacional de Colombia, Sede Palmira. 136 p.

9. Prager, M., Victoria, J., Sánchez De P, M., Gómez, E. \& Zamorano, A. (2000). El suelo y los abonos verdes una alternativa de manejo ecológico. Palmira. Universidad Nacional de Colombia, 18p. (serie de cuadernillos; no. 7).

10. Randhawa, P., Condron, L., Di, H., Sinaj, S. \& Mclenaghen, R. (2005). Effect of green manure addition on soil organic phosphorus mineralization. Nutrient Cycling in Agroecosystems, Vol.73. p 178-189.

11. Reyes J. T. (2001) Micelio externo de hongos micorrícicos arbusculares y su potencial influencia en la recuperación de suelos degradados en laderas del Cauca, Colombia. Tesis de maestría en Ciencias Agrarias. Universidad Nacional de Colombia, Sede Palmira. 88 p.
12. Sánchez De P., M. (1999). Endomicorrizas en algunos agroecosistemas de Colombia. Universidad Nacional de Colombia. Sede Palmira. Editorial Feriva, Cali. 227 p.

13. Sánchez De P., M. \& Gómez, E. (2003). Las micorrizas, los fijadores de nitrógeno y la economía en los agroecosistemas. Cuadernos ambientales No. 8. Universidad Nacional de Colombia Sede Palmira y Ministerio de Agricultura. p. 3-19.

14. Sánchez De P., M., Gómez, E., Muñoz, J. E., Barrios, E., Prager, M., Bravo, O., et al., (2007). Las endomicorrizas, expresión bioedáfica de importancia en el trópico. Universidad Nacional de Colombia. Sede Palmira. Editorial Feriva. $351 \mathrm{p}$.

15. Sánchez De P., M., Posada, R., Velásquez, D. \& Narváez, M. (2010ํ). Metodologías básicas para el trabajo con micorriza arbuscular y hongos formadores de micorriza arbuscular. Universidad Nacional de Colombia. Sede Palmira. $139 \mathrm{p}$.

16. Sánchez De P., M., Prager M., Ángel D. \& Sarria P. (2010b). Indicadores de sostenibilidad con enfoque agroecológico en agroecosistemas tropicales EN: LEÓN T. y ALTIERI M. Vertientes del pensamiento agroecológico: fundamentos y aplicaciones. Bogotá. Universidad Nacional de Colombia Instituto de Estudios Ambientales - IDEA y Sociedad Científica Latinoamericana de Agroecología (SOCLA). Opciones graficas editores Ltda. p. 247-269.

17. Sieverding, E. (1983). Vesicular - Arbuscular Mycorrhiza management in tropical agrosystems. GTZ, Republica Federal de Alemania. 372 p.

18. Torres, R. (2000). El papel del micelio externo de hongos que forman micorriza arbuscular asociado a barbechos mejorados en suelos degradados de ladera de Pescador (Cauca). Tesis de Maestría. Universidad Nacional de Colombia, Sede Palmira. 89 p.

19. USDA, (1996). Cover and green manure crop benefits to soil quality technical. Note No.1 (Abril). Recuperado de: http://soils.usda.gov/sqi/management/files/sq atn_1.pdf.

20. Zárate, L. M. (2006). Dinámica temporal en la formación de micelio externo de hongos micorrícico arbusculares (HMA) y su impacto en la formación de agregados estables al agua. Tesis de maestría en Ciencias Agrarias. Universidad Nacional de Colombia, Palmira. 108 p. 